\title{
Profiling Neutrophil-to-Lymphocyte Ratio Changes in Response to Nucleoside Analog Therapy for Chronic Hepatitis B Infection
}

\author{
MARNONETTE MARALLAG ${ }^{1,2}$, AMITKUMAR PATEL $^{2,3}$, MYUNGHAN CHOI $^{4}$, \\ MARK N. WONG ${ }^{5}$ and ANIL B. SEETHARAM ${ }^{2,5}$ \\ ${ }^{1}$ Internal Medicine, ${ }^{3}$ Gastroenterology, and ${ }^{5}$ Transplant and Advanced Liver Disease Center, \\ Banner University Medical Center, Phoenix, AZ, U.S.A.; \\ ${ }^{2}$ University of Arizona College of Medicine, Phoenix, AZ, U.S.A.; \\ ${ }^{4}$ Nursing and Health Care Innovation, Arizona State University, Tempe, AZ, U.S.A.
}

\begin{abstract}
Background/Aim: The neutrophil-lymphocyte ratio (NLR) has gained attention as an index of inflammation in patients with chronic hepatitis $B$ virus (HBV); however, changes with nucleoside analog therapy require investigation. Patients and Methods: We carried out a retrospective study identifying monoinfected $H B V$ patients initiated on therapy with NLR follow-up over 1 year. Biochemistries recorded at treatment initiation and 1 year included alanine aminotransferase (ALT), Model for End Stage Liver Disease (MELD) score, and NLR. Results: A total of 67 patients were initiated on therapy and had baseline characteristics including e-antigen (eAg) (50, 74.6\%) and cirrhosis (19, 28.4\%). On subgroup analysis among those with $\mathrm{HBV}$-associated cirrhosis, the NLR decreased over 1 year (3.08 \pm 0.39 vs. $1.77 \pm 0.18$, $p<0.001)$ as did MELD and ALT. Among the non-cirrhotic

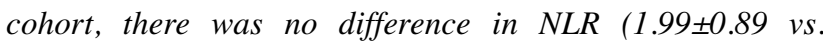
$2.14 \pm 1.03, p=0.134)$ despite a decrease in ALT. Conclusion: Nucleoside analog therapy in HBV cirrhosis is associated with a decrease in NLR over 1 year that tracks with changes of established indices of inflammation/global hepatic function.
\end{abstract}

The neutrophil-lymphocyte ratio (NLR) has gained attention as an index of inflammation and global hepatic synthetic function. The utility of NLR in disease pathogenesis and prognosis has

This article is freely accessible online.

Correspondence to: Anil B. Seetharam, Transplant and Advanced Liver Disease Center, Banner University Medical Center, 1441 North 12th Street, 2nd Floor, Phoenix, AZ 85006, U.S.A. E-mail: Anil.Seetharam@bannerhealth.com

Key Words: Chronic hepatitis B, neutrophil-to-lymphocyte ratio, cirrhosis, nucleoside analog therapy. been investigated in numerous clinical contexts including malignancy, cardiovascular disease and chronic liver disease $(1,2)$. Substantial increases in NLR have been reported in patients with acute liver failure when compared to hepatitis $\mathrm{B}$ virus (HBV)-infected patients without cirrhosis and healthy controls (3). Previous retrospective studies have also shown a significant direct correlation between NLR and increased severity of fibrosis (3) and frequency of decompensation (4). NLR has also been studied as a surrogate marker for global hepatic synthetic function and as an adjunct to the Model for End Stage Liver Disease (MELD) score; increased levels correlate with higher 1 - and 3-month mortality $(3,4)$.

HBV remains a significant cause of liver disease in the United States. Despite increased vaccination rates, chronic HBV prevalence has remained fairly consistent due to immigration of patients from areas of endemicity (5). Treatment of chronic HBV infection has been shown to reduce hepatic fibrosis, reverse cirrhosis, and reduce the development of hepatocellular carcinoma (HCC) (6). The role of NLR in chronic HBV pathogenesis, including necroinflammation, fibrosis and its role at defining sequela of HBV-related cirrhosis is not clearly defined.

Recently, NLR changes with peginterferon therapy for chronic HBV infection were evaluated and no significant difference in NLR before and after treatment was found (9). The primary aim of our investigation was to evaluate interval changes in NLR with nucleoside analog therapy in patients with and without HBV-related cirrhosis and further characterize its role in evaluating treatment responses.

\section{Patients and Methods}

This was a retrospective study of a cohort of 67 patients who were chronically monoinfected with HBV with clinical and laboratory follow-up over 1 year between 2010 to 2015 at the Outpatient Transplant and Advanced Liver Disease Center at Banner University 
Medical Center in Phoenix, Arizona (United States). For inclusion, the patients had to be HBsAg-positive, HBsAb negative, and receiving antiviral therapy for chronic $\mathrm{HBV}$ infection. Exclusion criteria included concurrent infection with hepatitis $\mathrm{C}$ virus. Baseline demographics prior to antiviral initiation were recorded including: age, ethnicity, body mass index (BMI), tobacco use history, eAg status, absence/presence of cirrhosis, and nucleoside analog therapy received. Cirrhosis was defined based on crosssectional imaging, biochemical testing or histological assessment with percutaneous biopsy. Biochemical parameters recorded/ calculated before and after one year of treatment included alanine aminotransferase (ALT), MELD score, and NLR. Variables were analyzed with paired-sample $t$-tests; differences with values of $p<0.05$ were considered significant.

\section{Results}

A total of 67 patients initiated on HBV therapy were identified, with a mean age of 51.2 (range=25-78) years and mean BMI $24.3 \pm 5.3 \mathrm{~kg} / \mathrm{m}^{2}$. Baseline demographics for the overall population are given in Table I. HBV monoinfected patients were initiated on specific oral antiviral therapy (Table I). Baseline demographics did not differ significantly between the cirrhotic and non-cirrhotic subgroups.

On subgroup analysis among patients with HBV-related cirrhosis $(n=19,28.3 \%)$ : NLR significantly decreased over 1 year follow-up $(3.08 \pm 0.39 v s .1 .77 \pm 0.18, p<0.001)$ similarly to the MELD score $(11.7 \pm 0.41 v s .7 .79 \pm 0.40, p<0.001)$ and ALT level (52.3 $\pm 9.69 v s .26 .6 \pm 4.76, p=0.006)$. Among the non-cirrhotic cohort $(n=48,71.6 \%)$ initiated on HBV antiviral therapy, no difference was seen in NLR after 1 year $(1.99 \pm 0.89 v s .2 .14 \pm 1.03, p=0.134)$ despite a significant decrease in ALT level (38.8 \pm 37.6 vs. 22.7 $\pm 13.8, p=0.003)$.

\section{Discussion}

Previous studies revealed that an increased preoperative NLR is predictive of increased tumor recurrence in patients with HBV-related liver disease complicated by HCC after liver transplantation (7). Similarly, a NLR $>2.5$ prior to interventional locoregional radiotherapy with radiofrequency ablation for HCC was significantly associated with tumor recurrence in patients infected with $\mathrm{HBV}$ (8). Although these previous studies reported prognostic value in NLR following treatment for $\mathrm{HBV}$-related $\mathrm{HCC}$, few examined changes in NLR related to initiation of antiviral treatment. In addition, temporal NLR trends associated with antiviral therapy with and without cirrhosis require further characterization.

Recently, NLR trends with peginterferon therapy were investigated (9). In our analysis of patients with liver disease related to $\mathrm{HBV}$, treatment with nucleoside analog therapy for 1 year was associated with a significant decrease in NLR, which in turn, tracked with changes of established indices of inflammation and global hepatic function, namely ALT and MELD score, respectively. Among patients with chronic
Table I. Baseline patient demographics.

\begin{tabular}{lccc}
\hline Demographic & $\begin{array}{c}\text { Total } \\
\text { population, } \\
\mathrm{N}=67\end{array}$ & $\begin{array}{c}\text { Cirrhosis } \\
\text { absent, } \\
\mathrm{N}=48\end{array}$ & $\begin{array}{c}\text { Cirrhosis } \\
\text { present, } \\
\mathrm{N}=19\end{array}$ \\
\hline Mean age, years & 51.2 & 50.8 & 52.9 \\
Male & $42(62.7 \%)$ & $28(58.3 \%)$ & $14(73.7 \%)$ \\
Ethnicity & $28(41.8 \%)$ & $21(43.8 \%)$ & $7(36.8 \%)$ \\
Caucasian & $27(40.3 \%)$ & $18(37.5 \%)$ & $9(47.4 \%)$ \\
Asian & $7(10.4 \%)$ & $7(14.6 \%)$ & $0(0 \%)$ \\
Hispanic & $5(7.5 \%)$ & $2(4.2 \%)$ & $3(15.8 \%)$ \\
Black & $24.3 \pm 5.3$ & $24.3 \pm 4.3$ & $26.9 \pm 7.0$ \\
Mean BMI, kg/m ${ }^{2}$ & & & \\
Tobacco use & $30(44.8 \%)$ & $22(45.8 \%)$ & $8(42.1 \%)$ \\
Current or past use & $37(55.2 \%)$ & $26(54.2 \%)$ & $11(57.9 \%)$ \\
Never & & & \\
eAntigen status & $17(25.4 \%)$ & $13(27.1 \%)$ & $4(21.1 \%)$ \\
Positive & $50(74.6 \%)$ & $35(72.9 \%)$ & $15(78.9 \%)$ \\
Negative & & & \\
Antiviral therapy & $36(53.7 \%)$ & $21(43.8 \%)$ & $15(78.9 \%)$ \\
Tenofovir & $19(28.4 \%)$ & $15(31.2 \%)$ & $4(21.0 \%)$ \\
Entecavir & $6(9.0 \%)$ & $6(12.5 \%)$ & $0(0 \%)$ \\
Lamivudine & $4(6.0 \%)$ & $4(8.3 \%)$ & $0(0 \%)$ \\
Adefovir & $2(3.0 \%)$ & $2(4.2 \%)$ & $0(0 \%)$ \\
Tenofovir/emtricitabine & & & \\
\hline
\end{tabular}

BMI, Body mass index.

hepatitis B without cirrhosis, no significant changes in NLR were seen despite an observed decrease in ALT level after 1 year of nucleoside analog therapy.

The present study was limited by its relatively small number of patients, particularly the cohort of cirrhotic patients. The retrospective nature of the study also limited observation of temporal trends in NLR during HBV infection. Nevertheless, the investigation does demonstrate that changes in NLR in HBV cirrhosis track similarly to other established indices of liver function in cirrhosis, while its sensitivity and specificity may be significantly compromised in those without advanced fibrosis. Further prospective studies are needed to evaluate temporal trends in NLR during HBV infection and its value as a biomarker of fibrosis and prognosis.

\section{References}

1 Gomez D, Farid S, Malik HZ, Young AL, Toogood GJ, Lodge JP and Prasad KR: Preoperative neutrophil-to-lymphocyte ratio as a prognostic predictor after curative resection for hepatocellular carcinoma. World J Surg 32(8): 1757-1762, 2008.

2 Ergelen M, Uyarel H, Altay S, Kul S, Ayhan E, Isik T, Kemaloglu T, Gul M, Sonmez O, Erdogan E and Turfan M: Predictive value of elevated neutrophil to lymphocyte ratio in patients undergoing primary angioplasty for ST-segment elevation myocardial infarction. Clin Appl Thromb Hemost 20(4): 427-432, 2014 
3 Chen L, Lou Y, Chen Y and Yang J: Prognostic value of the neutrophil-to-lymphocyte ratio in patients with acute-on-chronic liver failure. Int J Clin Pract 68(8): 1034-1040, 2014.

4 Zhang H, Sun Q, Mao W, Fan J and Ye B: Neutrophil-tolymphocyte ratio predicts early mortality in patients with HBVrelated decompensated cirrhosis. Gastroenterol Res Pract 2016 : 4394650, 2016.

5 Roberts H, Kruszon-Moran D, Ly KN, Hughes E, Igbal K, Jiles RB and Holmberg SD: Prevalence of chronic hepatitis B virus (HBV) infection in U.S. households: National Health and Nutrition Examination Survey (NHANES), 1988-2012. Hepatology 63(2): 388-397, 2016.

6 Tsochatzis EA, Bosch $\mathrm{J}$ and Burroughs AK: Liver cirrhosis. Lancet 383(9930): 1749-1761, 2014.

7 Xiao GQ, Liu C, Liu DL, Yang JY and Yan LN: Neutrophillymphocyte ratio predicts the prognosis of patients with hepatocellular carcinoma after liver transplantation. World $\mathrm{J}$ Gastroenterol 19(45): 8398-8407, 2013.
8 Tajiri K, Baba H, Kawai K, Minemura M, Yasumura S, Takahara $\mathrm{T}$ and Sugiyama T: Neutrophil-to-lymphocyte ratio predicts recurrence after radiofrequency ablation in hepatitis B virus infection. J Gastroenterol Hepatol 31(7): 1291-1299, 2016.

9 Le P, Liang K, Chang M, Hsu C, Chen Y, Lin C, Lin W, Lai M and Yeh C: Clinical predictors for neutrophil-to-lymphocyte ratio changes in patients with chornic hepatitis $\mathrm{B}$ receiving peginterferon treatment. In Vivo 31(4): 723-729, 2017.

Received August 1, 2017

Revised August 13, 2017

Accepted August 22, 2017 